\title{
AIDS, Human Capital and Development (Preliminary and Incomplete)
}

\author{
Rodolfo E. Manuelli* \\ Department of Economics Washington University in St. Louis \\ and Federal Reserve Bank of St. Louis \\ February 15, 2015
}

\begin{abstract}
In this paper I study a model in which the existence of a "disease environment" influences parental investment in early childhood human capital and individual schooling and on the job training decisions. The model is used to analyze the effect of HIV/AIDS on aggregate output per worker.

I use a calibrated version of the model to estimate the long run impact on output per worker of increasing life expectancy for individuals who have HIV/AIDS and reducing the rate of transmission of the disease in a subset of Sub-Saharan countries. I find that the effects on output per worker, the prevalence of the diseases and the growth rate of population can be substantial.
\end{abstract}

*I thank NSF for financial support. 


\section{Introduction}

There is no question that health improvements have contributed to the increase in the standard of living in many countries and, in particular, in less developed countries. It is also clear that there is a price tag associated with increasing health standards. Even though there is an intrinsic value associated with health, it is important from a public policy perspective to have some sense of the economic impact of interventions directed at improving the health status of a population.

The literature that measures the contribution of improved health to economic performance has not produced, so far, definitive results. The empirically based studies that regress output on some measure of health tend to find large economic returns from health improvements (e.g. Sachs (2003), Audibert (2010). Since, by its very nature, that approach is subject to the possibility of significant biases it is important to contrast the results with the predictions of more micro based models. The results from this approach are mixed. Some macro based models that allow for changes in population but model health improvements in a very stylized manner find that the economic impact of improving "health" is either relatively modest (e.g. see Weil (2007) and Ashraf, Lester and Weil (2008)) or directly negative (i.e. Acemoglu and Johnson (2007)). The findings of micro based studies (see the summary in

Bleakley (2010)) suggest that, in the case of some diseases (e.g. malaria and hookworm) the economic benefits of eradication are substantial.

One channel through which a disease can affect development that has not been thoroughly studied is through its impact on investments in human capital. Human capital is a complex stock that has both a quantity and 
a quality dimension. Existing studies that consider years of schooling a measure of quantity of human capital - as the only indicator were not designed to incorporate the effects of a disease environment on the quality dimensions of schooling as well as on investments training on-the-job. In some cases (e.g. Bleakley (2010a) and (2010b)), the evidence is consistent with the view that a change in the disease environment works its way through higher income by affecting the quantity and the quality of schooling and other forms of investment in human capital.

In this paper I study a simple model of human capital accumulation, both in the form of schooling and on-the-job training, that can be used to estimate the impact of changes in health conditions on human capital accumulation decisions and economic performance. The aim is to develop a model that is simple enough to be manageable and, at the same time, rich enough so that its implications can be disciplined by micro observations. In this draft, I concentrate on the case of a communicable disease that reduces life expectancy and, in that sense, I view the exercise as a stylized model of the macroeconomic impact of HIV/AIDS.

I consider two channels through which health influences human capital accumulation. First, an individual who has a given health condition can face a higher probability of permanent disability or death (e.g. if he is infected with HIV/AIDS). Second, a given health status can limit an individual's ability to supply effort (e.g. HIV/AIDS, malaria and other debilitating diseases). In addition, at the aggregate level, changes in the composition of the population can have a large effect on output per worker.

I calibrate the model using evidence from the U.S. to recover parameters 
of the human capital accumulation equation, and empirical micro studies of the impact of HIV/AIDS on income to estimate how health conditions are mapped into the parameters of the model. I then use the basic setting to study the benefits of changing the patterns of infection and the impact of ART drugs on output per worker. I compare the outcomes across steady states with those that correspond to a standard increase in productivity for a small sample of African economies.

Even though I view the exercise as very preliminary there are some interesting results. I find that reducing the incidence of malaria and the rate of transmission of HIV/AIDS to one half of their current values will result, in the long run, in an increase in output per worker between $25 \%$ and just over $40 \%$. An increase in life expectancy for infected individuals associated, for example, with better access to ART drugs, has similar effects.

The results show that the largest component of the increase in the stock of human capital associated with changes in HIV/AIDS prevalence is driven by increases in quality rather than quantity. Thus, models that ignore the quality dimension would tend to underestimate the beneficial effects of reducing the incidence of disease.

I also conduct a more traditional experiment: For each country I assume that productivity changes so that output increases by about $17 \%$. Unlike the case of the reduction in the incidence of HIV/AIDS, the role of schooling and quality of human capital are reversed, with the former accounting for about $60 \%$ of the increase in output. Thus, in this type of models, estimates of the role of schooling drawn from episodes of growth not associated with changes in the disease environment do not provide an accurate guide about 
the effect of improving health standards upon the quality-quantity mix of human capital.

\section{Africa: HIV/AIDS Indicators}

Even though there is consensus that HIV/AIDS is a serious health problem in many African countries, there is much less agreement about the fraction of individuals who are affected by this disease. In Table 1, I present some relatively homogeneous data on prevalence and incidence of HIV/AIDS for a small subset of African countries. ${ }^{1}$ The first two columns (from Oster (2012)) report UNAIDS estimates of prevalence and incidence of HIV/AIDS approximately over the 1985-2007 period. More recent prevalence data from UNAIDS (column labeled $P(15,49))$ suggests that the average estimates do not reflect in many cases the current situation. Oster (2012) also reports prevalence and incidence estimates inferred from mortality data (not shown here) for a subset of the countries and the values that she finds are smaller.

Irrespective of the preferred estimate, it is safe to conclude that there is a high degree of heterogeneity among African countries in the severity of the HIV/AIDS epidemic and that there are many countries in which a significant fraction of the population is HIV/AIDS positive. If HIV/AIDS has a large impact on productivity, the evidence is consistent with the view that the gains from eradication can be significant.

\footnotetext{
${ }^{1}$ See Table A.3 in the Appendix for the corresponding data for a larger set of countries.
} 


\begin{tabular}{|c|c|c|c|}
\hline \hline \multicolumn{4}{|c|}{ Table 1 } \\
\hline \hline \multicolumn{4}{|c|}{ HIV/AIDS: Prevalence and Incidence } \\
\hline \hline Country & $\bar{P}(15,49)$ & $I$ & $P(15,49)$ \\
Cameroon & 3.79 & 0.56 & 5.1 \\
Ghana & 1.35 & 0.20 & 1.9 \\
Kenya & 6.57 & 0.96 & 7.8 \\
Malawi & 8.79 & 1.31 & 11.9 \\
Mozambique & 5.94 & 1.01 & 12.5 \\
Zambia & 12.63 & 1.82 & 15.2 \\
Zimbabwe & 18.95 & 2.51 & 15.3 \\
\hline \hline Sources: $\bar{P}(15,49)$ and $I$ from Oster $(2012)$, \\
\hline \hline \multicolumn{2}{|c|}{$P(15,49)$ from UNAIDS (2008) } \\
\hline \hline
\end{tabular}

In interpreting the data it is necessary to take into account that all these measures reflect a mix of natural (or exogenous) disease conditions as well as behavioral responses and the impact of policies designed to mitigate or eliminate their effects.

\section{A Simple Model of Human Capital Accu- mulation}

Any model that succeeds in explaining the link between a particular health condition and individual decisions about how much human capital to acquire must take into account the effect of each health condition on morbidity which determines the cost of acquiring human capital and the rate at which 
it can be utilized - and on the probability of dying or becoming totally disabled. As a first approximation, I will model the mortality effect as a change in the constant instantaneous probability of dying. This amounts to assuming that lifetimes are close to exponential which is a rough approximation to the data. However, since the purpose of this paper is to describe a simple framework that can be used to match micro and macro evidence, this seems a natural first step.

The actual impact of health on economic conditions is probably dependent on the market structure and, it is possible, that markets are "more incomplete" is less developed countries ${ }^{2}$. However, a natural benchmark is the situation in which individuals are able to invest according to their potential as this provides an upper bound on the impact of diseases on human capital investment ${ }^{3}$, and it is this case that this paper studies.

In this context, consumption and human capital accumulation decisions can be studied separately. Thus, individuals maximize the present discounted value of income and then choose consumption according to that value.

It is useful to separate the optimal human capital accumulation problem in two phases: schooling and working. I identify the schooling period - whose length is determined endogenously — as being characterized by specialization in human capital accumulation. This extreme view, i.e. that children in

\footnotetext{
${ }^{2}$ See, however, the work of Townsend (XXXX) that shows that family structures come close to replicating the optimal allocation in some low income villages.

${ }^{3}$ For example, if due to incomplete markets a healthy individual cannot acquire any schooling, then a change in his health condition cannot possibly decrease the stock of human capital since he was at a corner. In general, very little can be said ex-ante about the impact of relaxing one condition in a second best situation.
} 
school do not work, can be shown to be inessential in closely related models. In future work, I will explore this issue in more detail. The working period is defined by the existence of a positive supply of labor. In what follows I work backwards to characterize the solution of the problem: I first study the problem faced by an individual who has already joined the labor force and then I discuss the choice of schooling.

\subsection{The Post-Schooling Problem: Susceptible Phase}

The amount of human capital that an individual wants to supply to the market depends on his productivity as well as his chances of dying or being permanently disabled. I first analyze the optimal labor supply/human capital accumulation problem of an individual who has been infected (say of AIDS) at age $p$ and whose endowment of labor is $v_{A} \in(0,1)$.

HIV/AIDS Positive. Let's denote the current level of human capital of such an individual by $h$. Let $R$ be that age at which the individual retires. Then, the present discounted value of income of an $a$ year old person, $V^{A}(h, a)$, is given by the solution to

$$
V^{A}(h, a)=\max _{n_{A}, h_{A}, x_{A}} E\left\{\int_{a}^{T^{\wedge} R} e^{-r(t-a)}\left[w\left(v_{A}-n_{A}(t)\right) h_{A}(t)-q x_{A}(t)\right] d a\right\}
$$

subject to

$$
\dot{h}_{A}(a)=z_{h}\left(n_{A}(a) h_{A}(a)\right)^{\gamma_{1}} x_{A}(a)^{\gamma_{2}}-\delta_{h} h_{A}(a),
$$

where the expectation is taken over the age of death, $T$. In this formulation $w$ is the wage rate per effective unit of human capital (which is different from the hourly wage rate), $v_{A}-n_{A}(a)$ is the amount of time allocated to actual 
production, and $\left(v_{A}-n_{A}(a)\right) h_{A}(a)$ is the effective human capital allocated to producing. Finally, $x(a)$ is the amount of market goods that are consumed in the process of on-the-job training. ${ }^{4}$

The key aspect of this formulation is that investment in human capital is rival to working and, hence, influenced by incentives beyond those that determine labor force participation.

The assumption of exponential lifetime with a maximum lifespan given by $\bar{T}$ (with $R \leq \bar{T}$ ) implies that the probability that an individual dies before age $t$ is

$$
P[T \leq t]=\frac{1-e^{-\lambda_{A} t}}{1-e^{-\lambda_{A} \bar{T}}}, \text { for } t \leq \bar{T}
$$

and life expectancy at age $a$ is just

$$
L^{A}(a)=\frac{1-e^{-\lambda_{A}(\bar{T}-a)}}{\lambda_{A}} .
$$

It follows that increases in the arrival rate $\lambda_{A}$ result in a lower life expectancy conditional on age.

A standard argument shows that the value function is the solution to the Hamilton-Jacobi-Bellman equation given by

$$
\begin{aligned}
r V^{A}(h, a)= & \max _{n, x}\left\{w\left(v_{A}-n\right) h-q x+\frac{\partial V^{A}}{\partial h}(h, a)\left[z_{h}\left(n h_{A}\right)^{\gamma_{1}} x^{\gamma_{2}}-\delta_{h} h\right)(1)\right. \\
& \left.+\frac{\partial V^{A}}{\partial a}(h, a)+\lambda_{A}\left[0-V^{A}(h, a)\right]\right\},
\end{aligned}
$$

\footnotetext{
${ }^{4}$ For example, if $1 / 10$ th of the normal working hours are devoted to learning/experimenting with different cultivation techniques, then $v_{A}-n(a)=9 / 10$, and $x(a)$ is the amount of resources (services of capital equipment, land and seeds in this example) used up in the learning process. Alternatively, this also captures the decision of a farmer that allocates $1 / 10$ th of his land to experimenting with new crops/techniques.
} 
with boundary condition

$$
V^{A}(h, R)=0
$$

A simple calculation shows that the value function is given by

$$
V^{A}(h, a)=w^{\frac{1-\gamma_{1}}{1-\gamma}} V_{0}^{A}\left(a ; v_{A}, \lambda_{A}\right)+w V_{1}^{A}\left(a ; v_{A}, \lambda_{A}\right) h
$$

where

$$
\begin{aligned}
& V_{1}^{A}\left(a ; v_{A}, \lambda_{A}\right)=v_{A} m\left(a ; \lambda_{A}\right), \\
& V_{0}^{A}\left(a ; v_{A}, \lambda_{A}\right)=(1-\gamma)\left[\frac{z_{h} \gamma_{1}^{\gamma_{1}} \gamma_{2}^{\gamma_{2}}}{p^{\gamma_{2}}}\right]^{\frac{1}{1-\gamma}} \int_{a}^{R} e^{-\rho\left(\lambda_{A}\right)(t-a)}\left(V_{1}^{A}(t)\right)^{\frac{1}{1-\gamma}} d t(2 \mathrm{~b})
\end{aligned}
$$

where $\gamma=\gamma_{1}+\gamma_{2}, \rho(\lambda)=r+\delta_{h}+\lambda$, and

$$
m\left(a ; \lambda_{A}\right)=\frac{1-e^{-\rho\left(\lambda_{A}\right)(R-a)}}{\rho\left(\lambda_{A}\right)} .
$$

In this context, two parameters summarize the impact of HIV/AIDS: the instantaneous probability of death (given by $\lambda_{A} d t$ ), and a measure and the effective endowment of labor of an infected individual $\left(v_{A} \leq 1\right)$.

Optimal behavior implies that the stock of human capital of an $a$ year old individual who was infected at age $p$ is given by,

$$
\begin{aligned}
h_{A}(a, p)= & e^{-\delta_{h}(a-p)}\left[h_{H}(p)+\left(z_{h} \gamma_{1}^{\gamma_{1}} \gamma_{2}^{\gamma_{2}}\right)^{\frac{1}{1-\gamma}}\right. \\
& \left.\left(\frac{w}{q}\right)^{\frac{\gamma_{2}}{1-\gamma}} \int_{p}^{a} e^{\delta_{h}(t-p)} V_{1}^{A}\left(t ; v_{A}, \lambda_{A}\right)^{\frac{\gamma}{1-\gamma}} d t\right],
\end{aligned}
$$

where $h_{H}(p)$ is the stock of human capital of a healthy individual who becomes infected at age $p$.

The stock of human capital is the sum of the undepreciated component of the stock at age $p, e^{-\delta_{h}(a-p)} h_{H}(p)$, and the investment made on the job 
which is given by

$$
\left(z_{h} \gamma_{1}^{\gamma_{1}} \gamma_{2}^{\gamma_{2}}\right)^{\frac{1}{1-\gamma}}\left(\frac{w}{q}\right)^{\frac{\gamma_{2}}{1-\gamma}} \int_{p}^{a} e^{\delta_{h}(t-a)} V_{1}^{A}\left(t ; v_{A}, \lambda_{A}\right)^{\frac{\gamma}{1-\gamma}} d t
$$

It follows that a higher real wage, as measured by $w / q$, results in higher stock of human capital though its impact on investment. Of particular interest in this case is the impact of $\lambda_{A}$ and $v_{A}$. Increases in $\lambda_{A}$, which correspond to higher mortality associated with HIV/AIDS, lower the marginal value of investing in human capital as measured by $V_{1}^{A}\left(a ; v_{A}, \lambda_{A}\right)$ and this, in turn, results in a lower level of the stock of human capital. Similarly, decreases in $v_{A}$-which is a measure of the total available time and, indirectly, of morbidity associated with HIV/AIDS - also reduce the stock of capital.

Even though the evolution of human capital is of interest in itself, it is the effective amount supplied to the market, $h_{A}^{e}(a, p)$, that determines the level of output. It can be shown that the time investment in on-the-job training is

$$
n_{A}(a) h_{A}(a, p)=\left(z_{h} \gamma_{1}^{1-\gamma_{2}} \gamma_{2}^{\gamma_{2}}\right)^{\frac{1}{1-\gamma}}\left(\frac{w}{q}\right)^{\frac{\gamma_{2}}{1-\gamma}} V_{1}^{A}\left(a ; v_{A}, \lambda_{A}\right)^{\frac{1}{1-\gamma}}
$$

and, since $h_{A}^{e}(a, p)=\left(v_{A}-n_{A}(a)\right) h_{A}(a, p)$, a simple calculation shows that effective human capital allocated to production is

$$
\begin{aligned}
& h_{A}^{e}(a, p)=v_{A} e^{-\delta_{h}(a-p)} h_{H}(p)+\left(z_{h} \gamma_{1}^{\gamma_{1}} \gamma_{2}^{\gamma_{2}}\right)^{\frac{1}{1-\gamma}}\left(\frac{w}{q}\right)^{\frac{\gamma_{2}}{1-\gamma}} \\
& {\left[\int_{p}^{a} e^{\delta_{h}(t-a)} V_{1}^{A}\left(t ; v_{A}, \lambda_{A}\right)^{\frac{\gamma}{1-\gamma}} d t-\gamma_{1} V_{1}^{A}\left(a ; v_{A}, \lambda_{A}\right)^{\frac{1}{1-\gamma}}\right] .}
\end{aligned}
$$

This formulation implies that

$$
\frac{\partial h_{A}^{e}}{\partial v_{A}}(a, p)>0, \frac{\partial h_{A}^{e}}{\partial \lambda_{A}}(a, p)<0, \text { and } \frac{\partial h_{A}^{e}}{\partial p}(a, p)>0 .
$$


As expected, a higher severity of HIV/AIDS as measured by lower $v_{A}$, higher $\lambda_{A}$ and lower age of infection (lower $p$ ) results in lower levels of human capital at any age. Moreover, the age-effective human capital supply is given by

$$
\begin{gathered}
\frac{\partial h_{A}^{e}(a, p)}{\partial a}=-\delta_{h} h_{A}^{e}(a, p)+\left(z_{h} \gamma_{1}^{\gamma_{1}} \gamma_{2}^{\gamma_{2}}\right)^{\frac{1}{1-\gamma}}\left(\frac{w}{q}\right)^{\frac{\gamma_{2}}{1-\gamma}} \\
{\left[V_{1}^{A}\left(a ; v_{A}, \lambda_{A}\right)^{\frac{\gamma}{1-\gamma}}\left(1+\frac{\gamma_{1}}{1-\gamma} e^{-\rho\left(\lambda_{A}\right)(R-a)}\right)-\gamma_{1} \delta_{h} V_{1}^{A}\left(a ; v_{A}, \lambda_{A}\right)^{\frac{1}{1-\gamma}}\right] .}
\end{gathered}
$$

To get a good sense of the qualitative properties of the the age-effective human capital supply consider first the case $\delta_{h}=0$. Then equation (6) implies that the supply of effective human capital is increasing in experience. For the calibration that I use $\delta_{h}$ is fairly small and qualitatively the age-supply profile is increasing until a few years before retirement. This property - which extends to the age supply of healthy individuals as well — plays an important role in determining the impact of changes in the disease environment upon output per worker. To see this consider two economies with the same $h_{A}^{e}(a, p)$ functions but different age distributions of the workforce. The model implies that the economy with the "older" population has a higher level of human capital. Thus, any change in a disease environment that reduces mortality will have the impact of making the average worker older and this, in turn, results in a higher level of aggregate human capital.

The model has implications for the level of income of an infected individual. Let $y_{A}(a, p)$ be the income level of an individual with HIV/AIDS who became infected at age $p$. Thus,

$$
y_{A}(a, p)=w h_{A}^{e}(a, p)-q x_{A}(a),
$$


and

$$
\begin{aligned}
y_{A}(a, p)= & w v_{A} e^{-\delta_{h}(a-p)} h_{H}(p)+w\left(z_{h} \gamma_{1}^{\gamma_{1}} \gamma_{2}^{\gamma_{2}}\right)^{\frac{1}{1-\gamma}}\left(\frac{w}{q}\right)^{\frac{\gamma_{2}}{1-\gamma}} \\
& {\left[v_{A} \int_{p}^{a} e^{\delta_{h}(t-a)} V_{1}^{A}\left(t ; v_{A}, \lambda_{A}\right)^{\frac{\gamma}{1-\gamma}} d t-\gamma V_{1}^{A}\left(a ; v_{A}, \lambda_{A}\right)^{\frac{1}{1-\gamma}}\right] . }
\end{aligned}
$$

As in the case of effective human capital, income is decreasing in $\lambda_{A}$ and increasing in $v_{A}$ and $p$.

HIV/AIDS Negative. The problem faced by a healthy individual who has already left school is similar to that of an infected person. There are two differences. First, a healthy individual has a higher effective supply of labor (i.e. $\left.1>v_{A}\right)$ and a lower instantaneous death probability $\left(\lambda_{H} d t<\right.$ $\left.\lambda_{A} d t\right)$. Second, this individual can become infected, and this happens with infinitesimal probability $\eta d t$.

In this case the relevant HJB equation is

$$
\begin{aligned}
r V^{H}(h, a)= & \max _{n, x}\left\{w(1-n) h-q x+\frac{\partial V^{H}}{\partial h}(h, a)\left[z_{h}\left(n h_{A}\right)^{\gamma_{1}} x^{\gamma_{2}}-\delta_{h} h\right] \quad(8)\right. \\
& \left.+\frac{\partial V^{H}}{\partial a}(h, a)+\lambda_{H}\left[0-V^{A}(h, a)\right]+\eta\left[V^{A}(h, a)-V^{H}(h, a)\right]\right\} .
\end{aligned}
$$

Relative to equation (1) — and other than the values of the disease environmentthe key difference is in the last term. It captures the "capital loss" associated to becoming infected. This is a risk that the individual takes into account when choosing strategies to increase his human capital while working.

It is possible to show that the solution to equation (8) is of the form

$$
V^{H}(h, a)=w^{\frac{1-\gamma_{1}}{1-\gamma}} V_{0}^{H}\left(a ; v_{A}, \lambda_{A}, \lambda_{H}, \eta\right)+w V_{1}^{H}\left(a ; v_{A}, \lambda_{A}, \lambda_{H}, \eta\right) h
$$


where

$$
\begin{aligned}
V_{1}^{H}\left(a ; v_{A}, \lambda_{A}, \lambda_{H}, \eta\right)= & \frac{1}{\rho\left(\lambda_{A}\right)}\left[\left(r+\delta_{h}+\lambda_{H}+\eta\right) m\left(a ; \lambda_{H}+\eta\right)-\right. \\
& \left.\eta e^{-\left(r+\delta_{h}+\lambda_{A}\right)(R-a)} m\left(a ; \lambda_{H}+\eta-\lambda_{H}\right)\right] .
\end{aligned}
$$

As in the case of healthy individuals, the key determinant of their human capital investment decisions is the term $V_{1}^{H}\left(a ; v_{A}, \lambda_{A}, \lambda_{H}, \eta\right)$. In order to describe the impact of the infection rate on the nature of the solution it is useful to describe the marginal value of human capital for an individual who is healthy and has zero probability of infection. It follows that $V_{1}^{H}\left(a ; v_{A}, \lambda_{A}, \lambda_{H}, 0\right)=m\left(a ; \lambda_{H}\right)>v_{A} m\left(a ; \lambda_{A}\right)$, as $v_{A}<1$ and $\lambda_{H}<\lambda_{A}$. The properties of $V_{1}^{H}\left(a ; v_{A}, \lambda_{A}, \lambda_{H}, \eta\right)$ are summarized in the following proposition:

Proposition 1 The marginal value of human capital to a healthy individual who is out of school, $V_{1}^{H}\left(a ; v_{A}, \lambda_{A}, \lambda_{H}, \eta\right)$, is such that:

$$
\begin{aligned}
& \text { 1. } V_{1}^{H}\left(a ; v_{A}, \lambda_{A}, \lambda_{H}, 0\right)>V_{1}^{H}\left(a ; v_{A}, \lambda_{A}, \lambda_{H}, \eta\right)>V_{1}^{A}\left(a ; v_{A}, \lambda_{A}\right) \\
& \text { 2. } \lim _{\eta \rightarrow \infty} V_{1}^{H}\left(a ; v_{A}, \lambda_{A}, \lambda_{H}, \eta\right)=V_{1}^{A}\left(a ; v_{A}, \lambda_{A}\right) \\
& \text { 3. } V_{1}^{H}\left(a ; v_{A}, \lambda_{A}, \lambda_{H}, \eta\right) \text { is decreasing in } \eta \text {. } \\
& \text { 4. } V_{1}^{H}\left(a ; v_{A}, \lambda_{A}, \lambda_{H}, \eta\right)-V_{1}^{A}\left(a ; v_{A}, \lambda_{A}\right) \text { is decreasing in } a .
\end{aligned}
$$

Proof. (See the Appendix)

Before discussing the implications of this result it is useful to describe the equilibrium investment in human capital of a healthy individual. Just as in 
the case of an infected person, time investments and goods investments are proportional and given by

$$
x_{H}(a)=\frac{\gamma_{2}}{\gamma_{1}} \frac{w}{p} n_{H}(a) h_{H}(a)
$$

and the investment of a healthy person relative to an infected individual is

$$
\frac{n_{H}(a) h_{H}(a)}{n_{A}(a) h_{A}(a)}=\left[\frac{V_{1}^{H}\left(a ; v_{A}, \lambda_{A}, \lambda_{H}, \eta\right)}{V_{1}^{A}\left(a ; v_{A}, \lambda_{A}\right)}\right]^{\frac{1}{1-\gamma}} .
$$

Equation (10) and the characterization of the marginal value of human capital in the proposition show that:

- Healthy individuals invest more in human capital than infected individuals, and that this gap is decreasing in the rate of infection.

- When an individual becomes infected equation (10) implies that this is accompanied by a decrease in investment.

- Age-earnings profiles of infected individuals are flatter than those of healthy individuals as they choose lower investment.

- The effect of infection on investment is decreasing in age: the older the individual at the time he becomes infected the smaller the adjustment.

\subsection{The Post-Schooling Problem: Non-Susceptible Phase}

Consider the decision of an individual who knows that his risk of infection until age $p^{*}$ is zero. This applies, for example, to young individuals who are non-drug users and who are not sexually active. The HJB equation for the 
period between leaving school and reaching age $p^{*}$ is given by

$$
\begin{aligned}
r V^{Y}(h, a)= & \max _{n, x}\left\{w(1-n) h-q x+\frac{\partial V^{Y}}{\partial h}(h, a)\left[z_{h}\left(n h_{A}\right)^{\gamma_{1}} x^{\gamma_{2}}-\delta_{h} h\right]\right. \\
& \left.+\frac{\partial V^{Y}}{\partial a}(h, a)+\lambda_{H}\left[0-V^{Y}(h, a)\right]\right\} .
\end{aligned}
$$

As before, the solution is of the form

$$
V^{Y}(h, a)=w^{\frac{1-\gamma_{1}}{1-\gamma}} V_{0}^{Y}\left(a ; v_{A}, \lambda_{A}, \lambda_{H}, \eta, p^{*}\right)+w V_{1}^{Y}\left(a ; v_{A}, \lambda_{A}, \lambda_{H}, \eta, p^{*}\right) h
$$

with the additional boundary condition

$$
V^{Y}\left(h, p^{*}\right)=V^{H}\left(h, p^{*}\right)
$$

Since the investment decision is totally dependent on the marginal value of human capital, $V_{1}^{Y}\left(a ; v_{A}, \lambda_{A}, \lambda_{H}, \eta, p^{*}\right)$, it is useful to study its properties. It can be shown that it is given by

$$
\begin{aligned}
V_{1}^{Y}\left(a ; v_{A}, \lambda_{A}, \lambda_{H}, \eta, p^{*}\right)= & \frac{w}{\rho\left(\lambda_{H}\right)}\left[1-e^{-\rho\left(\lambda_{H}\right)\left(p^{*}-a\right)}\right] \\
& +w V_{1}^{H}\left(p^{*} ; v_{A}, \lambda_{A}, \lambda_{H}, \eta\right) e^{-\rho\left(\lambda_{H}\right)\left(p^{*}-a\right)}
\end{aligned}
$$

Note that if $p^{*}=R$ then the individual has zero risk of infection. In this case, $V_{1}^{Y}\left(a ; v_{A}, \lambda_{A}, \lambda_{H}, \eta, R\right)=V_{1}^{H}\left(a ; v_{A}, \lambda_{A}, \lambda_{H}, 0\right)$. It follows that increases in $p^{*}$ - the minimum age at which an individual becomes susceptible-increases the return on human capital. Formally (and suppressing the arguments to simplify the notation)

$$
\frac{\partial V_{1}^{Y}}{\partial p^{*}}(a)=w e^{-\rho\left(\lambda_{H}\right)\left(p^{*}-a\right)}\left[1+\frac{\partial V_{1}^{H}}{\partial a}\left(p^{*}\right)-\rho\left(\lambda_{H}\right) V_{1}^{H}\left(p^{*}\right)\right] .
$$

However, it is easy to check that optimal behavior in the susceptible stage requires that for any $a \geq p^{*}$

$$
\frac{\partial V_{1}^{H}}{\partial a}(a)=\rho\left(\lambda_{H}+\eta\right) V_{1}^{H}(a)-1-\eta V_{1}^{A}(a)
$$


which implies that

$$
\frac{\partial V_{1}^{Y}}{\partial p^{*}}(a)=w e^{-\rho\left(\lambda_{H}\right)\left(p^{*}-a\right)} \eta\left[V_{1}^{H}(a)-V_{1}^{A}(a)\right]>0
$$

given that $V_{1}^{H}(a)-V_{1}^{A}(a)$.

Finally, it follows that the positive effect gets smaller as the individual ages and it disappears at $a=p^{*}$. These changes as a function of age suggest that interventions that change some parameters of the disease environment will have differential effects on individuals depending on their age at the time of the change.

\subsection{The Schooling Period}

During the period between age 6 and $6+s$, I assume that the average individual is at school full time. In this model, the length of the schooling period, $s$, is endogenous. I assume following Manuelli and Seshadri (2014) that the technology to accumulate human capital is the same throughout an individual's life except that, to simplify the calculations, I set the depreciation rate to zero during the schooling period.

It turns out that it is necessary to distinguish between children who are born with HIV/AIDS — and hence who can die during the period before they reach age $p^{*}$ — and children who are born healthy and, in this version of the model, have zero probability of becoming infected until age $p^{*}$.

\subsubsection{Healthy Children}

If an individual starts school with a stock $h_{E}$ of early childhood human capital and allocates $100 \%$ of his time to building human capital through 
formal schooling, his optimal choice of schooling quality is the solution to the following problem

$$
\max _{x_{s}} \int_{6}^{6+s} e^{-\left(r+\lambda_{H}\right)(t-6)} x_{s}(t) d t+e^{-r s} V^{Y}(h(6+s), 6+s)
$$

subject to

$$
\dot{h}(t)=z_{s} h(t)^{\gamma_{1}} x_{s}(t)^{\gamma_{2}},
$$

where, as before, the risk of death appears as a higher discount rate. An analysis of this problem (see the Appendix for details) shows that the optimal $x_{s}(t)$ satisfies

$$
x_{s}(t)=x_{s} e^{\frac{r+\lambda_{H}}{1-\gamma_{2}}(t-6)}, \text { for } t \in[6,6+s]
$$

and that the stock of human capital at the end of the schooling period is

$$
h(6+s)=\left[h_{E}^{1-\gamma_{1}}+\frac{\left(1-\gamma_{1}\right) z_{s}}{\phi} x_{s}^{\gamma_{2}}\left(e^{\phi s}-1\right)\right]^{\frac{1}{1-\gamma_{1}}}, \text { where } \phi=\frac{\gamma_{2}\left(r+\lambda_{H}\right)}{1-\gamma_{2}} .
$$

Equation (13) shows that higher quality of schooling — as measured by the initial level of material inputs, $x_{s}$ - and longer schooling — as measured by $s$ - increase the level of human capital.

I assume that the stock of early childhood human capital depends positively on the amount of resources allocated to children (e.g. expenditures on health, nutrition, and living quarters) according to

$$
h_{E}=h_{B} x_{E}^{v}
$$

The cost of schooling inputs and resources for early childhood at age $6+s$ is simply

$p_{E} x_{E} e^{r s}+\int_{6}^{6+s} e^{\left(r+\lambda_{H}\right)(6+s-t)} x_{s} e^{\frac{\left(r+\lambda_{H}\right)}{1-\gamma_{2}}(t-6)} d t=e^{\left(r+\lambda_{H}\right) s}\left[p_{E} x_{E}+x_{s} \frac{e^{\phi s}-1}{\phi}\right]$. 
Then, optimal choice of schooling quality and quantity by a healthy individual is the solution to

$$
\max _{x_{E}, x_{s}, s} V^{Y}(h(6+s), 6+s)-e^{\left(r+\lambda_{H}\right) s}\left[p_{E} x_{E}+x_{s} \frac{e^{\phi s}-1}{\phi}\right],
$$

subject to

$$
h(6+s) \leq H\left(x_{E}, x_{s}, s\right) \equiv\left[\left(h_{B} x_{E}^{v}\right)^{1-\gamma_{1}}+\frac{\left(1-\gamma_{1}\right) z_{s}}{\phi} x_{s}^{\gamma_{2}}\left(e^{\phi s}-1\right)\right]^{\frac{1}{1-\gamma_{1}}}
$$

To do: Describe FOCS.

\subsubsection{Infected Children}

There are several differences between the problem faced by infected children. First, they face a higher probability of death $\left(\lambda_{A}>\lambda_{H}\right)$ and that, to capture morbidity, their endowment of time is lower. This implies that the technology to produce human capital is

$$
\dot{h}(t)=z_{s}^{A} h(t)^{\gamma_{1}} x_{s}(t)^{\gamma_{2}}, \text { where } z_{s}^{A}=z_{s} \zeta_{A}^{\gamma_{1}} \text { for } \zeta_{A} \in(0,1]
$$

Second, the value of human capital when they finish schooling is given by $V^{A}(h, 6+s)$. Thus the analogues of equations (12) and (13) are

$$
\max _{x_{E}, x_{s}, s} V^{A}(h(6+s), 6+s)-e^{\left(r+\lambda_{A}\right) s}\left[p_{E} x_{E}+x_{s} \frac{e^{\phi_{A} s}-1}{\phi_{A}}\right],
$$

subject to

$$
\begin{aligned}
h(6+s) \leq H^{A}\left(x_{E}, x_{s}, s\right) & \equiv\left[\left(h_{B} x_{E}^{v}\right)^{1-\gamma_{1}}+\frac{\left(1-\gamma_{1}\right) z_{h}^{A}}{\phi_{A}} x_{s}^{\gamma_{2}}\left(e^{\phi_{A} s}-1\right)\right]^{\frac{1}{1-\gamma_{1}}}, \\
& \text { with } \phi_{A}=\frac{\gamma_{2}\left(r+\lambda_{A}\right)}{1-\gamma_{2}} .
\end{aligned}
$$




\section{The Distribution of the Population}

In this section I derive the steady state distribution of the population. It is convenient to separately keep track of the number of healthy individuals, those who have HIV/AIDS. Since the impact on human capital depends on the age at which an individual is infected, I also need to keep track of the joint distribution of the health status and age of infection.

To see the factors that affect the distribution consider the healthy population. Let $N^{H}\left(a, p^{*} ; t\right)$ be the number of healthy individuals of age less than $a$ (for $a \geq p^{*}$ ) at time time $t$. This population changes because some people age (and are no longer in the age category), individuals who reach age $p^{*}$ now belong to this population, and some people die or become infected. Let the mass of people in between ages $p^{*}$ and $a$ be denoted by $M^{H}\left(a, p^{*} ; t\right)$. Formally, $M^{H}\left(a, p^{*} ; t\right)=N^{H}\left(a, p^{*} ; t\right)-N^{H}\left(p^{*}, p^{*} ; t\right)$. It follows that the evolution of this population in this group satisfies

$$
\frac{\partial M^{H}}{\partial a}\left(a, p^{*} ; t\right)+\frac{\partial M^{H}}{\partial a}\left(a, p^{*} ; t\right)=-\left(\lambda_{H}+\eta\right) M^{H}\left(a, p^{*} ; t\right)+\frac{\partial N^{H}}{\partial a}\left(p^{*}, 0 ; t\right) .
$$

For healthy individuals younger than $p^{*}$-and hence free from infection risk - the relevant equations is

$$
\frac{\partial N^{H}}{\partial a}(a, 0 ; t)+\frac{\partial N^{H}}{\partial a}(a, 0 ; t)=-\lambda_{H} N^{H}(a, 0 ; t)+B^{H}(t)
$$

where $B^{H}(t)$ is the number of people born at time $t$.

I concentrate on the balanced growth stationary distribution and assume that population is growing at a constant rate. This implies that $N^{H}(a, 0 ; t)=$

$\Phi^{H}(a, 0) G(t)$, with $\dot{G}(t)=g G(t)$ for some $g$ that will depend on fertility and 
mortality parameters. In addition, I assume that births in this category are given by

$$
B^{H}(t)=\beta^{H} \bar{N}^{H}(t)+\beta_{H}^{A} \bar{N}^{A}(t)
$$

where $\beta_{K}^{J}$ is the number of births per population in category $J$ that are born in health status $K$, and $\bar{N}^{J}(t)$ is the total population with health status $J$ at time $t$, for $J, K \in\{H, A\}$

Given the balanced growth assumption it follows that

$$
\bar{N}^{J}(t)=\bar{N}^{J} e^{g t}, \quad J \in\{H, A\}
$$

Given these assumptions it follows that $\Phi^{H}(a, 0)$ satisfies

$$
\dot{\Phi}^{H}(a, 0)=-\left(\lambda_{H}+g\right) \Phi^{H}(a, 0)+\bar{B}^{H}
$$

where

$$
\bar{B}^{H}=\beta^{H} \bar{N}^{H}+\beta_{H}^{A} \bar{N}^{A}
$$

Since $N^{H}(a, 0 ; t)$ is a cumulative distribution must satisfy $N^{H}(0,0 ; t)=0$, and this implies that $\Phi^{H}(a, 0)$ is

$$
\Phi^{H}(a, 0)=\frac{\bar{B}^{H}}{\lambda_{H}+g}\left[1-e^{-\left(\lambda_{H}+g\right) a}\right] \text { for } a \leq p^{*} .
$$

Next, consider the solution to equation (14). As in the previous case we conjecture that it has the form

$$
M^{H}\left(a, p^{*} ; t\right)=C(a) e^{g t}
$$

Then, the function $C(a)$ must satisfy

$$
\dot{C}(a)=-\left(\lambda_{H}+\eta+g\right) C(a)+\bar{B}^{H} e^{-\left(\lambda_{H}+g\right) p^{*}}
$$


and the unique solution that satisfies the boundary condition $C\left(p^{*}\right)=0$ is

$$
C(a)=\frac{\bar{B}^{H} e^{-\left(\lambda_{H}+g\right) p^{*}}}{\lambda_{H}+\eta+g}\left[1-e^{-\left(\lambda_{H}+\eta+g\right)\left(a-p^{*}\right)}\right] .
$$

The evolution of the healthy population is then given by $N^{H}(a ; t)=$ $\Phi^{H}(a) e^{g t}$ with

$$
\Phi^{H}(a)= \begin{cases}\frac{\bar{B}^{H}}{\lambda_{H}+g}\left[1-e^{-\left(\lambda_{H}+g\right) a}\right] & \text { for } a \leq p^{*} \\ \frac{\bar{B}^{H} e^{-\left(\lambda_{H}+g\right) p^{*}}}{\lambda_{H}+\eta+g}\left[1-e^{-\left(\lambda_{H}+\eta+g\right)\left(a-p^{*}\right)}\right]+\frac{\bar{B}^{H}}{\lambda_{H}+g}\left[1-e^{-\left(\lambda_{H}+g\right) p^{*}}\right] & \text { for } a>p^{*}\end{cases}
$$

Since the maximum lifespan is $\bar{T}$, consistency requires that

$$
\begin{aligned}
\bar{N}^{H}= & \Phi^{H}(\bar{T})=\frac{\bar{B}^{H} e^{-\left(\lambda_{H}+g\right) p^{*}}}{\lambda_{H}+\eta+g}\left[1-e^{-\left(\lambda_{H}+\eta+g\right)\left(\bar{T}-p^{*}\right)}\right] \\
& +\frac{\bar{B}^{H}}{\lambda_{H}+g}\left[1-e^{-\left(\lambda_{H}+g\right) p^{*}}\right]
\end{aligned}
$$

The other category of interest is the population with HIV/AIDS. As indicated before, from the point of view of their supply of human capital it is important to take into account the age of infection. Let $N^{A}(a, p ; t)$ be the number of people of age less than $a$ who were infected at age $p$, with $p \in\{0\} \cup\left[p^{*}, \bar{T}\right]$. Then, the evolution of this population for $p \neq 0$ satisfies

$$
\frac{\partial N^{A}}{\partial a}(a, p ; t)+\frac{\partial N^{A}}{\partial a}(a, p ; t)=\eta N^{H}(a ; t)-\lambda_{A} N^{A}(a, p ; t), \text { for } a \geq p
$$

with boundary condition $N^{A}(p, p ; t)=0$.

If $p=0$ (this corresponds who individuals born with HIV/AIDS) the appropriate expression is

$$
\frac{\partial N^{A}}{\partial a}(a, 0 ; t)+\frac{\partial N^{A}}{\partial a}(a, 0 ; t)=-\lambda_{A} N^{A}(a, 0 ; t)+B^{A}(t),
$$

where, paralleling the previous formulation

$$
B^{A}(t)=\beta_{A}^{A} \bar{N}^{A}(t)
$$


since I assume that only HIV/AIDS infected individuals can give birth to HIV/AIDS newborns. The relevant boundary condition is $N^{A}(0,0 ; t)=0$, and the stationary distributions are

$$
\begin{gathered}
\Phi^{A}(a, p)=\frac{\bar{B}^{H} e^{-\left(\lambda_{H}+\eta+g\right) p}}{\lambda_{A}+g}\left[1-e^{-\left(\lambda_{A}+g\right)(a-p)}\right] \\
\Phi^{A}(a, 0)=\frac{\beta_{A}^{A} \bar{N}^{A}}{\lambda_{A}+g}\left[1-e^{-\left(\lambda_{A}+g\right) a}\right] .
\end{gathered}
$$

The infected population (as a share of the total) between the ages of 0 and $a$ is given by

$$
\int_{p^{*}}^{a} \Phi^{A}(a, p) d p+\Phi^{A}(a, 0)=\bar{N}^{A}(a)
$$

while the healthy population (again as share of the total) is

$$
\Phi^{H}(a)=\bar{N}^{H}(a)
$$

Consistency requires that

$$
\max \left[0, \int_{p^{*}}^{\bar{T}} \Phi^{A}(\bar{T}, p) d p\right]+\Phi^{A}(\bar{T}, 0)=\bar{N}^{A}
$$

and

$$
\bar{N}^{A}+\bar{N}^{H}=1
$$

Equations (20), (17) and (21) determine the growth rate of population, $g$, and the fractions of the population that are healthy, $\bar{N}^{H}$, and infected, $\bar{N}^{A}$.

\section{Equilibrium}

I concentrate on the steady state. Given the interest rate $r$ and aggregate productivity $z$, the capital effective labor, $\kappa$, satisfies

$$
r+\delta_{k}+g=z F_{k}(\kappa, 1)
$$


the wage rate is

$$
w=z F_{n}(\kappa, 1)
$$

and output per worker is

$$
y=z F(\kappa, 1) \bar{h}^{e}
$$

where $\bar{h}^{e}$ is average - over all the age groups - human capital per worker.

Assume that $6+s<p^{* 5}$ then

$$
\begin{aligned}
\bar{h}^{e}= & {\left[\int_{6+s}^{R} h_{H}^{e}(a) \frac{\partial \Phi^{H}(a)}{\partial a} d a+\int_{p}^{R} h_{A}^{e}(a, 0) \frac{\partial \Phi^{A}(a, 0)}{\partial a} d a\right.} \\
& \left.+\int_{p^{*}}^{R}\left[\int_{p}^{R} h_{A}^{e}(a, p) \frac{\partial \Phi^{A}(a, p)}{\partial a} d a\right] d p\right] /[\bar{N}(R)-\bar{N}(6+s)]
\end{aligned}
$$

where $\bar{N}(a)=\bar{N}^{H}(a)+\bar{N}^{A}(a)$ is the total workforce.

The first term in equation (22) corresponds to the effective human capital supplied by healthy individuals while the last two reflect the supply of human capital by HIV/AIDS infected persons.

The definition of aggregate effective human capital per worker shows that improvements in the disease environment affect output per worker through two channels. First, they result in increases in the human capital for each type of worker and this is the effect studied in Section 3. Second, they change the composition of the population both between healthy and infected agents and along the age dimension. More specifically, since the human capital model implies that middle age workers supply more effective labor than younger individuals, and change that reduces mortality increases the share of the workforce accounted for by older workers and this, in turn, results in higher average human capital.

\footnotetext{
${ }^{5}$ Since the intent is to apply the model to Sub-Saharan African economies this conditions is satisfied as $s \approx 4-6$ and $p^{*}=14$.
} 
Unlike the models discussed by Young (2005), Weil (2007) and Ashraf et. al. (2009), I assume that savings adjust so as to keep the capital-human capital ratio equal to the user cost of capital. If the population growth rate does not change in response to changes in health, the capital-human capital ratio is unchanged and the change in output is completely determined by changes in joint distribution of health status and wage of the labor force.

\section{Calibration}

In order to use the model to quantitatively evaluate the impact of changes in the disease environment it is necessary to assign values to the parameters of the model. We use a year as a basic period.

The parameters of the human capital accumulation technology, $\gamma_{1}, \gamma_{2}, z_{h}$, are taken from Manuelli and Seshadri (2014). I take $\bar{T}$ - the maximum life span - to be 120 . The parameter $\lambda_{A}$ is associated with the life expectancy conditional on having HIV/AIDS. The model implies that life expectancy at age $a\left(a \geq p^{*}\right)$ is given by

$$
L^{A}(a)=\frac{1-e^{\lambda_{A}(\bar{T}-a)}}{\lambda_{A}} .
$$

If the life expectancy of a 20 year old individual with HIV/AIDS is 10 years, then the estimate of $\lambda_{A}$ is close to 0.10 . There is additional evidence that suggests that this is a reasonable value. Salomon (2006) presents data on incidence, prevalence and mortality estimates corresponding to the year 2000. Since

$$
\frac{\text { Mortality }}{100,000}=\lambda_{A} P
$$

Using a simple average of male and females values Salomon's data implies 


\begin{tabular}{llll}
\hline \hline Concept & AFRO D & AFRO E & World \\
\hline \hline$\lambda_{A}$ & 0.10 & 0.094 & 0.08 \\
\hline
\end{tabular}

According to Salomon, Gakidou and Murray (undated) a good approximation to the distribution of death times conditional on infection is given by the Weibull distribution. Using this distribution, the probability that an infected individual will die before $\tau$ years after infection is

$$
W(\tau)=1-\exp \left\{-\varkappa_{0} \tau^{\varkappa_{1}}\right\} .
$$

If this probability is to match the probability according to the exponential distribution, it must be the case that

$$
\varkappa_{0} \tau^{\varkappa_{1}-1}=\lambda_{A} .
$$

Since for the reported parameters the mean of the Weibull distribution is 10, the estimate of $\lambda_{A}$ according to this criterion would be 0.08 . Finally, if instead of matching the cumulative probability I chose to match the mean, then the estimate of $\lambda_{A}$ is 10 . Young (2005) presents data on cumulative survival rates. He shows that the probability of surviving more than 10 years after infection is approximately 0.40 . Thus, another estimate of $\lambda_{A}$ is given by

$$
\lambda_{A}=-\frac{\ln (0.40)}{10}=0.091
$$

It seems that even though it is not clear that the data come from a steady state distribution - and much less from an exponential - a reasonable estimate of $\lambda_{A}$ is somewhere between 0.08 and 0.10 , with values for high infection areas closer to the upper bound. 
In addition to its impact on life expectancy HIV/AIDS can potentially reduce an individuals ability to work. The estimates are controversial and in many cases emphasize the loss of income in the last few years. I consider two possible values of $v_{A}, 0.01$ and 0.05 which corresponds to a decrease in the endowment of labor of $1 \%$ and $5 \%$ respectively.

The fertility behavior of HIV/AIDS individuals relative to non-HIV/AIDS agents is a controversial subject and the empirical literature has not settled the issues related to the overall impact of the disease on birth rates. Young (2005) argues that an increase in the prevalence of HIV/AIDS lowers fertility. More recent analysis by Fortson (2009) and Juhn et. al. (2009) are consistent with the view that birth rates for infected women are about $20 \%$ lower and that the prevalence of HIV/AIDS has little impact on the fertility of healthy women ${ }^{6}$. Thus, as a first approximation, I assume

$$
\beta^{A}=0.80 * \beta^{H} .
$$

Let $\beta_{A}^{A}=m \beta^{A}$ and $\beta_{H}^{A}=(1-m) \beta^{A}$. The parameters $\beta^{H}$ and $m$ need to be determined

For each country, I have data on incidence and prevalence of AIDS. Let this be indicated by $I$ and $P$, respectively. Then, the model implies that

$$
P\left(p^{*}, a\right)=\bar{N}^{A}(a)-\bar{N}^{A}\left(p^{*}\right)
$$

corresponds to prevalence between the ages of $p^{*}$ and $a$. Incidence is given by

$$
I=\beta_{A}^{A} \bar{N}^{A}+\eta \int_{p^{*}}^{\bar{T}} \frac{\partial \Phi^{H}(a)}{\partial a} d a .
$$

\footnotetext{
${ }^{6}$ See also the findings and the discussion in Kalemli-Ozcan (2009a) and (2009b).
} 
The growth rate of the economy, g, given the other parameters, must solve,

$$
\int_{p^{*}}^{\bar{T}} \Phi^{A}(\bar{T}, p) d p+\Phi^{A}(\bar{T}, 0)+\Phi^{H}(\bar{T})=1 .
$$

Finally, life expectancy at age $a\left(a \leq p^{*}\right)$ is given by

$$
L(a)=L^{H}(a) \frac{\partial \Phi^{H}(a)}{\partial a}+L^{A}(a) \frac{\partial \Phi^{A}(a, 0)}{\partial a}
$$

where life expectancy of healthy and infected individuals is given by

$$
\begin{gathered}
L^{H}(a)=\frac{1}{\lambda_{H}}\left[1-e^{-\lambda_{H}\left(p^{*}-a\right)}\right]+\frac{1}{\lambda_{H}} \frac{\eta+\lambda_{A}}{\eta+\lambda_{H}}\left[1-e^{-\left(\lambda_{H}+\eta\right)\left(\bar{T}-p^{*}\right)}\right]+ \\
\frac{\eta}{\lambda_{A}} \frac{1}{\lambda_{A}-\left(\lambda_{H}+\eta\right)}\left[e^{-\lambda_{A}\left(\bar{T}-p^{*}\right)}-e^{-\left(\lambda_{H}+\eta\right)\left(\bar{T}-p^{*}\right)}\right], \text { for } a \leq p^{*}
\end{gathered}
$$

and

$$
L^{A}(a)=\frac{1}{\lambda_{A}}\left[1-e^{-\lambda_{A}(\bar{T}-a)}\right], \text { for } a \leq p^{*} .
$$

Equations (24) - (27) jointly determine $\left(\lambda_{H}, \eta, \beta^{H}, m\right)$

\section{AIDS and Development: A Preliminary Ex- ercise}

In this section I report the implications of the model when $R=\bar{T}=\infty$ since this is not a bad approximation given the parameters. The details of the calibration are in the Appendix. The basic strategy is simple: I compute the equilibrium in each country using its population parameters (from Table A.2) but I assume that technological parameters (other than TFP) are the same across countries. I then choose for each country a level of TFP so that the model's predictions for output per worker coincide with the estimate in 
version 6.3 of Summers-Heston Dataset. I assume that $v_{A}=0.95$ which captures the idea that an infected person losses about $5 \%$ of the available working days because of his health status.

\subsection{The Role of Human Capital}

Before reviewing the predictions of the model for the impact of changes associated with health policy, it is useful to understand the channels through which disease and economics interact. In Table 2 I present estimates of some measures of quality and quantity of human capital. The column labeled $\bar{h}^{e} / s$ is an index of the amount of human capital per worker supplied to the market per year of formal schooling. Even though the units themselves do not have an interpretation, the indicator is comparable across countries and I normalized Ghana's level to one.

\begin{tabular}{|c|c|c|}
\hline \hline \multicolumn{3}{|c|}{ Table 2. Human Capital and Schooling } \\
\hline \hline Country & $y$ & $\bar{h}^{e} / s$ \\
\hline \hline Cameroon & 1.98 & 1.15 \\
Ghana & 1 & 1.00 \\
Kenya & 1.26 & 0.93 \\
Malawi & 0.82 & 0.80 \\
Mozambique & 1.24 & 0.83 \\
Zambia & 1.37 & 0.89 \\
Zimbabwe & 1.47 & 0.90 \\
\hline \hline
\end{tabular}




\subsection{Disease: Reducing HIV/AIDS Transmission}

The first exercise that I consider models the situation in which, through behavioral or policy changes, the rate of transmission of HIV/AIDS can be cut in half. To be precise, the experiment takes the basic estimates from the calibrated version of the model and recomputes the equilibrium with a value of $\eta$ which is half the calibrated value. ${ }^{7}$

In Table 3, I present the predictions of the model (in the form of percentage changes relative to the base case) for the changes in output per worker, $\Delta y$, levels of schooling, $\Delta s$, an indicator of prevalence of HIV/AIDS, $P$, and the index of average human capital per year of schooling.

\begin{tabular}{|c|c|c|c|c|}
\hline \multicolumn{6}{|c|}{ Table 3. Lower Transmission Rate (\%) } \\
\hline \hline Country & $\Delta y$ & $\Delta P$ & $\Delta s$ & $\Delta\left(\bar{h}^{e} / s\right)$ \\
\hline \hline Cameroon & 9.2 & -2.5 & 1.8 & 7.7 \\
Ghana & 4.0 & -1.0 & 1.2 & 2.9 \\
Kenya & 14.2 & -4.1 & 4.4 & 9.5 \\
Malawi & 19.5 & -5.8 & 13.6 & 3.4 \\
Mozambique & 21.4 & -6.2 & 9.4 & 10.5 \\
Zambia & 23.8 & -7.3 & 11.6 & 10.2 \\
Zimbabwe & 24.3 & -7.7 & 8.6 & 15.1 \\
\hline \hline
\end{tabular}

The effects decreasing HIV/AIDS transmission on output per worker can be quite large. In countries where HIV/AIDS does not affect a large fraction

\footnotetext{
${ }^{7}$ Even though this is a significant reduction it seems within the boundaries of what is possible. McNeil (2010) reports that a vaginal gel used by women reduced the infection rate by $39 \%$ on average and by $54 \%$ among those women who used the gel consistently.
} 
of the population (e.g. Ghana) the gains are modest. At the other end, in countries with a significant fraction of HIV/AIDS individuals in the population the gains in output per worker exceed $30 \%$ in the long run. As a rough approximation, the model implies that a $1 \%$ decrease in the prevalence of HIV/AIDS through the decrease in the transmission rate increases long run output on average by $4.0 \%$, with the impact slightly lower for high prevalence areas.

The results in Table 3 suggest that even though average schooling increases, this changed is dwarfed by the increase in human capital per year of schooling. The decrease in the probability of infection qualitatively has the same effect as an increase in effective life expectancy which corresponds to an increase in the utilization rate of human capital. This results in more schooling but also higher quality and more investment in on-the-job training.

As a separate exercise I considered reducing the mother to child infection rate to one half of the calibrated value. For this set of countries the impact on all variables was minimal. The largest effect on output per worker occurs in Zimbabwe and it amounts to an increase in output per worker of just above $0.1 \%$. For that reason, I do not report the results of the exercise.

\subsection{Disease: Increasing Life Expectancy for Infected Individuals}

I view a decrease in $\lambda_{A}$ as a simple way of estimating the effect of the wider availability of ART drugs. Table 4 shows the effects of doubling life expectancy - halving the value of $\lambda_{A}$ - for individuals that become infected with HIV/AIDS. The results are in some dimensions similar to those cor- 
responding to lower infection rates: changes in output per worker (in the steady state) are large for countries in which HIV/AIDS affects a significant fraction of the population. The largest increases in human capital occur in the quantity (schooling) dimension, as the effective amount of human capital per year of schooling, $\bar{h}^{e} / s$, displays smaller increments.

\begin{tabular}{|c|c|c|c|}
\hline \multicolumn{3}{|c|}{ Table 4. Higher Life Expectancy (\%) } \\
\hline \hline Country & $\Delta y$ & $\Delta s$ & $\Delta\left(\bar{h}^{e} / s\right)$ \\
\hline \hline Cameroon & 10.8 & 1.9 & 7.9 \\
Ghana & 4.5 & 1.3 & 2.8 \\
Kenya & 16.5 & 4.8 & 9.0 \\
Malawi & 23.5 & 16.1 & 0.8 \\
Mozambique & 26.2 & 11.0 & 8.7 \\
Zambia & 29.9 & 13.7 & 7.7 \\
Zimbabwe & 32.5 & 9.5 & 18.1 \\
\hline \hline
\end{tabular}

\subsection{Development}

In this section I explore the consequences of a $3 \%$ increase in the marginal product of labor. In the context of this model this does not correspond to the conventionally measured real wage since the latter is given by the product of the marginal product of labor and the stock of human capital supplied to the market. Since human capital is not a simple function of schooling as quality is endogenous, adjustments for schooling do not map conventional measures of hourly wages into marginal product. This change in the marginal product roughly corresponds to a $2 \%$ increase in true TFP and yields increases in output per worker of about $17 \%$. 
The results are in Table 5 .

\begin{tabular}{|c|c|c|c|}
\hline \hline \multicolumn{3}{|c|}{ Table 5. Development (\% change) } \\
\hline \hline Country & $\Delta y$ & $\Delta s$ & $\Delta\left(\bar{h}^{e} / s\right)$ \\
\hline \hline Cameroon & 16.9 & 7.6 & 2.2 \\
Ghana & 17.0 & 11.5 & -1.0 \\
Kenya & 16.9 & 9.5 & -0.1 \\
Malawi & 16.9 & 14.1 & -5.4 \\
Mozambique & 16.9 & 9.3 & -0.5 \\
Zambia & 16.9 & 9.8 & -1.6 \\
Zimbabwe & 16.9 & 11.3 & -1.5 \\
\hline \hline
\end{tabular}

There are several interesting observations. First, the quality dimension of human capital appears less responsive to increases in productivity than to changes in the disease environment as they account for a smaller fraction of the increase in output per worker. Second, even an across the board productivity change has differential impacts when it comes to schooling between malarious and non malarious individuals. In all cases the response of years of education to a change in the marginal product of capital is larger for the poorer and less educated individuals. Finally, the increase in schooling reduces labor force participation and this implies that output per capita does not increase as much as output per worker. The differences vary by country and appear to be driven by demographics and the initial level of schooling. 


\section{Conclusion and Directions for Further Re- search}

In this paper I developed a model that can be used to evaluate the effect of changes in a disease environment on output per worker. The version that I discuss corresponds to a stylized model of the effect of HIV/AIDS on development though the human capital channel. The analysis so far has been restricted to steady states and, hence, I only capture very long run effects of health improvements.

I find that for a small sample of African economies the potential effects on output per worker of halving the rate of transmission of HIV/AIDS and doubling life expectancy associated with the infection are large and range somewhere between $20 \%$ and $35 \%$. The model suggests that improvements in the disease environment are not neutral and they affect human capital investment decisions differentially.

Given the preliminary nature of the exercise, it seems safe to view the results as suggesting, at best, orders of magnitude. The basic message is that incorporating explicitly a decision on how much to invest in human capital - both quantity and quality - yields estimates of the gains associated with large changes in a disease environment that are significantly larger than those found in the literature. Given that the analysis only looks at steady states it is not possible to use the model to describe the short run effects, although closely related models display long convergence times (of the order of over forty years).

The second important message is that improvements in a disease envi- 
ronment look different from similar improvements in TFP from the point of view of the necessary investments. In the model, a better disease environment significantly affects the quality composition of human capital and, in particular, the amount of investments made in the post-schooling period (quality dimension). This result is quite different form the predictions of the model when the shock to the economy is an increase in productivity. The latter has a large impact on schooling and smaller effects on the quality dimension.

Much work remains to be done since the model, as implemented in this draft, has limitations. First, it assumes a stylized distribution of lifetimes. This was done to facilitate the computation and to highlight the role of the disease parameters in influencing human capital choices. More realistic representations of the disability/death effects are feasible but require more complex models and are more computationally demanding. Second, the assumption of homogeneous agents (at least ex-ante) is not necessary but removing it simply makes the quantitative exercise more computationally demanding.

The model also abstracts away from capital market imperfections that may limit an individual's ability to invest in human capital for reasons unrelated to the disease environment, which is one the factors highlighted by Acemoglu and Johnson (2009). Incorporating this type of imperfection is feasible but requires a more careful modeling of the role of the family as a substitute to the market. I also considered the case in which the interest rate is constant. However, it is possible to endogeneize it in a way that incorporates the effect of population growth and the number of children along the lines of Manuelli and Seshadri (2009). 
The paper is silent about the welfare effects of these changes for two reasons. First, it ignores the cost side of the interventions. Second, it only looks at steady states in which case welfare comparisons are meaningless.

Last, but not least, I model the changes in the disease environment as exogenous. Even if it is reasonable to view changes in the price of drugs and other inputs that are relevant to the spread of a disease as exogenous, the rate at which they are adopted certainly is not ${ }^{8}$. Therefore, I view my estimates as "potential" gains rather than a prediction associated with any given policy intervention.

\footnotetext{
${ }^{8}$ For a good analysis of endogenous choices that affect the incidence of HIV/AIDS see
} Greenwood et. al. (2010) 


\section{Appendix}

Calibration The common calibrated values are

\begin{tabular}{llllllllll}
\hline \hline \multicolumn{1}{c}{ Table A.1: Common Parameters } \\
\hline \hline$\gamma_{1}$ & $\gamma_{2}$ & $\delta_{h}$ & $z_{s}$ & $z_{h}$ & $h_{B}$ & $r$ & $\alpha$ & $\delta_{k}$ \\
\hline .63 & .30 & .018 & .33 & .37 & 1.64 & .0531 & .33 & .071
\end{tabular}

In Table A.2 I present the estimates of the relevant population parameters when I use a measure of life expectancy that is an approximation to life expectancy conditional on reaching age 5 . The column labeled $\hat{g}$ is the internally consistent population growth rate, that is, the growth rate implied by the model. 


\section{Table A.2: Calibrated Population Parameters}

\begin{tabular}{|c|c|c|c|c|c|}
\hline Country & $L(5)$ & $\lambda_{H}$ & $\beta^{H}$ & $m$ & $\eta$ \\
\hline Angola & 54 & .018 & .061 & .149 & .003 \\
\hline Burkina Faso & 58 & .017 & .053 & .138 & .002 \\
\hline Cameroon & 59 & .016 & .039 & .218 & .006 \\
\hline Cent. Af. Rep. & 58 & .016 & .042 & .219 & .007 \\
\hline Congo & 58 & .017 & .059 & .166 & .002 \\
\hline Cote d'Ivoire & 60 & .016 & .038 & .327 & .004 \\
\hline Ethiopia & 63 & .016 & .054 & .231 & .002 \\
\hline Ghana & 64 & .015 & .031 & .216 & .002 \\
\hline Kenya & 60 & .016 & .042 & .239 & .010 \\
\hline Malawi & 56 & .016 & .052 & .196 & .016 \\
\hline Mali & 58 & .017 & .057 & .181 & .002 \\
\hline Mozambique & 58 & .015 & .045 & .152 & .016 \\
\hline Namibia & 65 & .013 & .026 & .296 & .019 \\
\hline Nigeria & 58 & .017 & .048 & .186 & .004 \\
\hline Sierra Leone & 54 & .018 & .061 & .125 & .002 \\
\hline South Africa & 55 & .015 & .026 & .210 & .002 \\
\hline Tanzania & 56 & .016 & .048 & .207 & .007 \\
\hline Zambia & 52 & .017 & .052 & .183 & .020 \\
\hline Zimbabwe & 46 & .019 & .037 & .272 & .018 \\
\hline
\end{tabular}

The values of Incidence and Prevalence for an extended set of countries 


\begin{tabular}{|c|c|c|c|}
\hline \multicolumn{4}{|c|}{ Table A.3 } \\
\hline \multicolumn{4}{|c|}{ HIV/AIDS: Prevalence and Incidence } \\
\hline Country & $\bar{P}(15,49)$ & $I$ & $P(15,49)$ \\
\hline Angola & 1.00 & 0.17 & 2.1 \\
\hline Burkina Faso & 1.87 & 0.25 & 1.6 \\
\hline Cameroon & 3.79 & 0.56 & 5.1 \\
\hline Cent. Af. Rep. & 4.43 & 0.66 & 6.3 \\
\hline Congo & 4.37 & 0.56 & 3.9 \\
\hline Cote d'Ivoire & 4.24 & 0.57 & 3.9 \\
\hline Ethiopia & 1.67 & 0.25 & 2.1 \\
\hline Ghana & 1.35 & 0.20 & 1.9 \\
\hline Kenya & 6.57 & 0.96 & 7.8 \\
\hline Malawi & 8.79 & 1.31 & 11.9 \\
\hline Mali & 0.86 & 0.14 & 1.5 \\
\hline Mozambique & 5.94 & 1.01 & 12.5 \\
\hline Namibia & 8.12 & 1.32 & 15.3 \\
\hline Nigeria & 1.98 & 0.31 & 3.1 \\
\hline Sierra Leone & 0.88 & 0.15 & 1.7 \\
\hline South Africa & 8.95 & 1.49 & 18.1 \\
\hline Tanzania & 5.92 & 0.82 & 6.2 \\
\hline Zambia & 12.63 & 1.82 & 15.2 \\
\hline Zimbabwe & 18.95 & 2.51 & 15.3 \\
\hline \multicolumn{4}{|c|}{ Sources: $\bar{P}(15,49)$ and $I$ from Oster $(2012)$, } \\
\hline$P(15,4$ & rom UN & $\mathrm{S}$ & 08) \\
\hline
\end{tabular}




\section{References}

[1] Acemoglu, D. and S. Johnson, (2007), "Disease and Development: The Effect of Life Expectancy on Economic Growth," Journal of Political Economy, Vol. 115, No. 6, pp: 925-985.

[2] Ashraf, Q., A. Lester and D. Weil, (2009), "When Does Improving Health Raise GDP?" NBER Macroeconomics Annual.

[3] Ashraf, Q., G. Fink and D. Weil, (2010), "Evaluating the Effects of Large Scale Health Interventions in Developing Countries: The Zambian Malaria Initiative," NBER working paper 16069, (June)

[4] Audibert, M., P. Combes Motel, and A. Drabo, (2010), "Global Burden of Disease and Economic Growth," CERDI, Etudes et Documents, E 2010.36, (December)

[5] Barofsky, J., C. Chase, T. Anekwe and F. Farzadfar, (2011), "The Economic Effects of Malaria Eradication: Evidence from and Intervention in Uganda," PGDA Working Paper No. 70, Harvard University

[6] Barreca, (2009), "The Long-Term Impact of In Utero and Postnatal Exposure to Malaria," Journal of Human Resources, (forthcoming).

[7] Bhattacharyya, S., (2009), "Root Causes of African Underdevelopment," Journal of African Economies, 10.

[8] Bleakley H., (2009), "When Does Improving Health Raise GDP? Comments on Ashraf, Lester and Weil," NBER Macroeconomics Annual. 
[9] Bleakley, (2010a), "Malaria Eradication in the Americas: A Retrospective Analysis of Childhood Exposure" American Economic Journal: Applied, April, 2(2):1-45

[10] Bleakley, H, (2010b), "Health, Human Capital and Development," working paper.

[11] Bundy D., M, Kremer, H. Bleakley, M. Jukes and E. Miguel, (2009), "Deworming and Development: Asking the right Questions, Asking the Questions Right," PLOS, Vol. 3, Issue 1.

[12] Cutler, D, W. Fung, M. Kremer, M. Singhal and T. Vogl, (2010), "Earlilife Malaria Exposure and Adult Outcomes: Evidence from Malaria Eradication in India," American Economic Journal: Applied Economics, Vol. 2, pp: 72-94.

[13] Fortson, J., (2009), "HIV/AIDS and Fertility," American Economic Journal: Applied Economics, 1(3), July, pp: 170-194.

[14] Fortson, J., (forthcoming), "Mortality Risk and Human Capital Investment: The Impact of HIV/AIDS in Sub-Saharan Africa," Review of Economics and Statistics.

[15] Greenwood, J. P. Kircher and M. Tertilt, (2010), "An Equilibrium Model of the African HIV/AIDS Epidemic," working paper.

[16] Gollin, D. and C. Zimmermann, (2007), "Malaria: Disease Impacts and Long-Run Income Differences," IZA DP No. 2997, (August). 
[17] Jayachandran S. and A. Lleras-Muney, (2009), "Life Expectancy and Human Capital Investments: Evidence from Maternal Mortality Declines," The Quarterly Journal of Economics, February, pp: 349-397.

[18] Juhn, C., S. Kalemli-Ozcan, and B. Turan, (2009), "HIV and Fertility in Africa: First Evidence from Population Based Surveys," working paper.

[19] Kalemli-Ozcan, S., (2009a), "HIV and Fertility Revisited," working paper.

[20] _ _ _ _ _ _, (2009b), "AIDS, 'Reversal' of the Demographic Transition and Economic Development: Evidence from Africa," working paper.

[21] Korenromp, E, (2005), "Malaria Incidence at Country Level for year 2004: Proposed Estimates and Draft Report," World Health Organization, Roll Back Malaria.

[22] Lucas, A., (2010), "Malaria Eradication and Educational Attainment: Evidence from Paraguay and Sri Lanka," American Economic Journal Applied, April, 2(2).

[23] Manuelli, R. and A. Seshadri, (2009), "Explaining International Fertility Differences," Quarterly Journal of Economics, Vol. 124, pp: 771-807.

[24] Mc Neil G., (2010), "Advance on AIDS Raises Questions as Well as Joy," New York Times, July 26, 2010. 
[25] Meng, X. and N. Qian, (20006), "The Long Run Health and Economic Consequences of Famine on Survivors: Evidence from China's Great Famine," CEPR Discussion Paper 5989.

[26] Oster, E., (2012), "Routes of Infection: Exports and HIV Incidence in Sub-Saharan Africa," Journal of the European Economic Association, October,10(5), pp:1025-1058.

[27] Sachs, J, (2003), "Institutions Don't Rule: Direct Effects of Geography on per Capita Income," NBER working paper No. 9490.

[28] Salomon, J., E. Gakidou and C. J. L. Murray, (undated), "Methods for Modeling the HIV/AIDS Epidemic in Sub-Saharan Africa," GPE Discussion Paper Series No. 3, EUG/GPE/EBD. (Available at the WHO website).

[29] Salomon, J. (2006), "Global Burden of HIV/AIDS in the Year 2000," $\mathrm{EIP} / \mathrm{GPE} / \mathrm{EBD}$, processed.

[30] Weil, D., (2007), "Accounting for the Effect of Health on Economic Growth," The Quarterly Journal of Economics, Vol. 122 (3), pp: 12651306.

[31] Weil, D. W., (2010), "The Impact of Malaria on African Development over the Longue Durée," Brown University Working Paper.

[32] World Health Organization (2004), "Global Burden of Disease 2004 Update: Disability Weights for Diseases and Conditions," WHO processed. 
[33] World Health Organization (2008), "HIV/AIDS Epidemiological Surveillance Report for the WHO African Region: 2007 update," Regional Office for Africa.

[34] Young, A., (2005), "The Gift of the Dying: The tragedy of AIDS and the Welfare of Future African Generations," The Quarterly Journal of Economics, 